\title{
Study of Antibiotic Sensitivity and Resistance Pattern of Bacterial Isolates in Intensive Care Unit Setup of a Tertiary Care Hospital
}

\author{
Sneha S Savanur ${ }^{1}$, Hemamalini Gururaj ${ }^{2}$
}

\begin{abstract}
Objective: To evaluate the antibiotic sensitivity and resistance pattern in an intensive care unit (ICU) setting of a tertiary care hospital. Materials and methods: A cross-sectional, retrospective study was conducted for a period of 1 month in October 2017 on a total of 195 patients who were admitted to ICU of tertiary care hospital. The culture and sensitivity pattern of clinical isolates from blood, urine, sputum, endotracheal tube (ET) aspirate, catheter sites, and wound swabs were analyzed. Positive cultures were segregated and their antibiotic sensitivity testing was performed under the guidelines of clinical and laboratory standard institute (CLSI).

Results: Of the total $195 \mathrm{ICU}$ admissions, cultures were sent for 167 cases. Of which 127 patients were culture positive and 40 cases were culture negative. Isolated bacteria were mostly gram-negative bacilli, of which Escherichia coli was (18.6\%), Acinetobacter (14.5\%), Klebsiella (11.6\%), Pseudomonas (9.8\%), and Proteus (1.74\%). Among the gram-positive organisms, coagulase negative staphylococcus (CoNS) (15.6\%) was most commonly isolated followed by Streptococcus (2.32\%). Fungal growth was also seen in 26 (15.11\%) samples. Samples that grew organisms were blood ( $n=48)$, sputum ( $n=17)$, urine $(n=39)$, ET aspirate $(n=40)$, pus $(n=11)$, catheter $(n=4)$, ear swab $(n=2)$, and stool $(n=1)$.

Conclusion: Gram-negative bacterial infections are increasing in ICUs, leading to inappropriate selection of antibiotics. Hence, antibiotic sensitivity and resistance pattern in a hospital setup has to be studied so as to guide the treating consultant to initiate empirical antibiotics in critical cases. Keywords: Antibiogram, Antibiotic, Culture, Intensive care unit, Resistance, Sensitivity.

Indian Journal of Critical Care Medicine (2019): 10.5005/jp-journals-10071-23295
\end{abstract}

\section{INTRODUCTION}

Antibiotics have served as the corner stone of modern medicine. Emergence of antibiotic resistance is a worldwide public health problem and a threat to mankind. ${ }^{1}$ In India, the burden of infectious disease is highest among the world; and recent reports showed the inappropriate and irrational use of antimicrobial agents against the diseases led to increase in the development of antimicrobial resistance (AMR). ${ }^{2}$ Besides poor financial conditions, inadequate infrastructure, high burden of disease, and unregulated sales of cheap antibiotics have amplified the crisis of AMR in India.,4

Bacterial infections are a frequent cause of hospitalization, and particularly nosocomial infections are more common in critical care settings. ${ }^{5}$ Globally the emergence of antibiotic resistance and limited availability of treatment options present an increasing challenge for the management of bacterial infections worldwide. Rate of nosocomial infections range from 5\% to $30 \%$ among ICU patients. The increased risk of infection is associated with severity of patient illness, length of exposure to invasive devices and procedures, increased patient contact with healthcare personnel, and length of stay in hospital. Over the past 15-20 years, infection control practices and new antimicrobial development have primarily targeted control and treatment of infections caused by gram-positive organisms. ${ }^{6-9}$ Recently the incidence of infections caused by gram-negative bacteria in ICU has risen, and the lack of available treatment options against some multi-drug-resistant (MDR) strains is alarming. Infections caused by MDR gram-negative organisms are associated with high morbidity and mortality. ${ }^{10}$ Hence, careful adherence to infection control and infection treatment guidelines helps to improve patient outcome and reduce hospital cost.

\begin{abstract}
1,2 Department of General Medicine, SDM College of Medical Sciences and Hospital, Dharwad, Karnataka, India

Corresponding Author: Hemamalini Gururaj, Department of General Medicine, SDM College of Medical Sciences and Hospital, Dharwad, Karnataka, India, Phone: +91 9740781723, e-mail: hemag972@gmail. com

How to cite this article: Savanur SS, Gururaj H. Study of Antibiotic Sensitivity and Resistance Pattern of Bacterial Isolates in Intensive Care Unit Setup of a Tertiary Care Hospital. Indian J Crit Care Med 2019;23(12):547-555.
\end{abstract}

Source of support: Nil

Conflict of interest: None

In this study, we analyze the pattern of antibiotic sensitivity and resistance based on the results of various cultures of microbial specimens from admitted patients. Information obtained may be crucial as a reference for pathogen identification and selection of empirical antibiotic therapy in our ICU setup.

\section{Materials and Methods}

Retrospective observational study conducted in a teaching tertiary care hospital during October 2017, a total of 195 adult patients admitted to ICU in this study period were included. Patients in whom cultures were not sent for testing were excluded. Data were collected from MRD of the hospital including patient identity, diagnosis, comorbidities, source of infection, results of microbial culture, antibiotic sensitivity and resistance pattern, antibiotic use, duration of stay in hospital, and clinical outcome. Of the total 195 ICU admissions, cultures were sent for 167 cases, of which 127 patients were culture positive and 40 cases were

o The Author(s). 2019 Open Access This article is distributed under the terms of the Creative Commons Attribution 4.0 International License (https://creativecommons. org/licenses/by-nc/4.0/), which permits unrestricted use, distribution, and non-commercial reproduction in any medium, provided you give appropriate credit to the original author(s) and the source, provide a link to the Creative Commons license, and indicate if changes were made. The Creative Commons Public Domain Dedication waiver (http://creativecommons.org/publicdomain/zero/1.0/) applies to the data made available in this article, unless otherwise stated. 
culture negative. In few cases, culture sample was sent from more than one site based on patient's clinical requirement. Isolated bacteria were mostly gram-negative organisms like E. coli (18.6\%), Acinetobacter (14.5\%), Klebsiella (11.6\%), Pseudomonas (9.8\%), and Proteus (1.74\%). Among the gram-positive organisms, CoNS (15.6\%) was most commonly isolated followed by Streptococcus (2.32\%). Fungal growth was also seen in 26 (15.11\%) samples. Specimens on which grew organisms were blood ( $n=48)$, sputum ( $n=17)$, urine $(n=39)$, ET aspirate $(n=40)$, pus $(n=11)$, central venous catheter tip $(n=4)$, ear swab $(n=2)$, and stool $(n=1)$.

\section{Results}

During the study period, a total of 195 cases were admitted to the medical ICU, of which 165 cases were sent for culture and sensitivity. A total of 127 cases had growth of organisms, which were tested for sensitivity pattern by standard laboratory methods, remaining 40 cases were culture negative. Among the culture grown cases, 100 samples were gram-negative and 46 were gram-positive organisms and 26 were positive for fungal growth as depicted in Figure 1.

The positive isolates are obtained from the following samples: blood $(n=48)$, sputum $(n=17)$, urine $(n=39)$, ET aspirate $(n=40)$, pus $(n=11)$, catheter $(n=4)$, stool $(n=1)$, ear swab $(n=2)$, and vaginal swab ( $n=1)$ (Fig. 2). CoNS is the most frequent isolate from

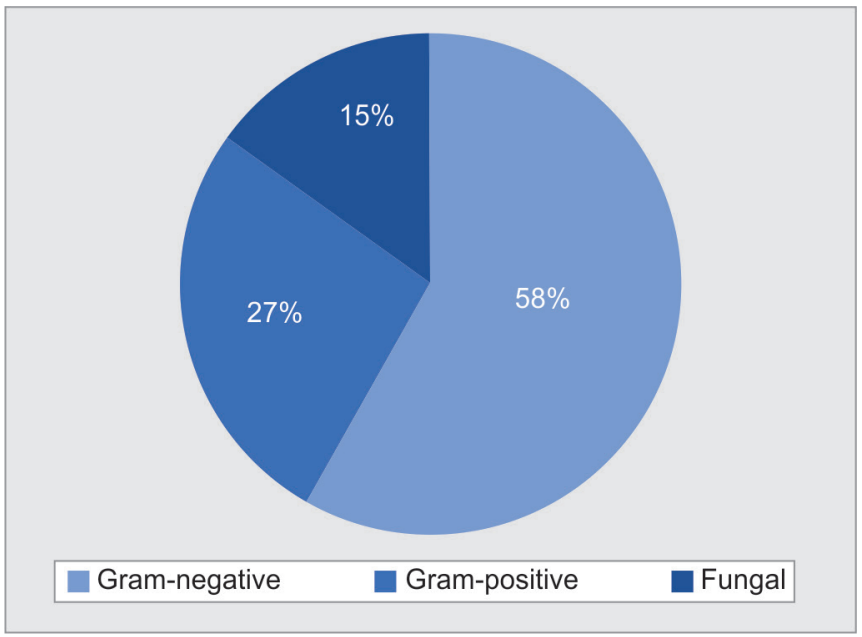

Fig. 1: Gram's staining and organisms isolated blood culture, E. coli and fungal growth from urine culture, and Klebsiella and Acinetobacter from ET secretions.

E. coli $(18.6 \%)$ was the most common organism isolated, followed by Acinetobacter (14.5\%), Klebsiella (11.6\%), Pseudomonas $(9.8 \%)$, and Proteus (1.74\%). Among the gram-positive organisms, CoNS $(15.6 \%)$ was the most common organism followed by Streptococcus (2.32\%). In all, 26 samples, i.e., (15.11\%) were positive for fungal growth (Table 1).

E. coli was most sensitive to colistin $(96.8 \%)$, followed by tigecycline (78.12\%), nitrofurantoin (71.8\%), imipenem (68.75\%), and meropenem (68.75\%) (Fig. 3). Similarly Figures 4 to 6 depict sensitivity pattern of Pseudomonas and Klebsiella, respectively.

Acinetobacter showed highest sensitivity to colistin (68\%) followed by tigecycline (64\%) (Fig. 7). Staphylococcus showed 100\% sensitivity to tigecycline and nitrofurantoin. Similarly Table 2 depicts the sensitivity pattern of other isolated organisms.

E. coli, Acinetobacter, Pseudomonas, Proteus, and Enterobacter showed resistance to cephalosporins and piperacillin-tazobactam. Resistance to colistin was observed more in Proteus, and CoNS Staphylococcus showed $100 \%$ resistance to vancomycin and clindamycin, as depicted in Table 3.

\section{Discussion}

Antibiotic resistance is an emerging problem in critically ill cases, which affects prognosis and survival of the patients. It also results in prolonged stay in hospital, increasing the cost of treatment. ${ }^{11-13}$

In our study, of the 167 cases sent, $76 \%$ were culture positive compared to $46.4 \%$ by Chakravarthi et al. ${ }^{14}$ Among these, gramnegative accounted for $58 \%$, gram-positive were $27 \%$, and fungal growth was yielded in 15\% of samples (Fig. 1).

Samples sent for culture were blood $(n=48)$, urine $(n=39)$, ET aspirate $(n=40)$, central venous catheter tips $(n=4)$, sputum $(n=17)$, and pus ( $n=11)$ (Fig. 2).

The most common organisms isolated in our study were E. coli (18.6\%), Klebsiella (11.6\%) Acinetobacter (14.5\%), and Pseudomonas $(9.8 \%)$. This is comparable to other studies where gram-negative organisms were most commonly isolated. ${ }^{10}$ Among gram-positive, CoNS was the most common organism isolated (15.6\%). Fungal growth was also seen in $15.11 \%$ samples (Table 1 ).

In Asian countries including India, most of the isolates obtained from ICU patients are gram-negative organisms such as $E$. coli,

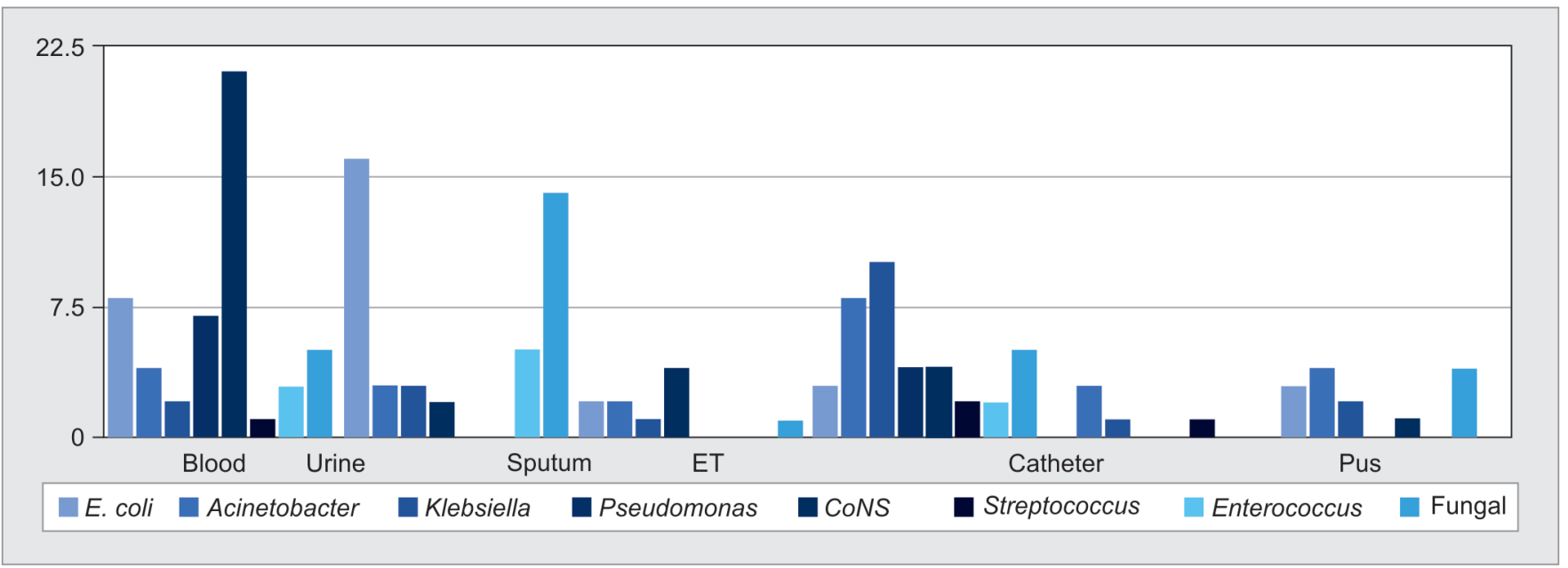

Fig. 2: Type of culture sample and organism isolated 
Klebsiella, and Acinetobacter followed by gram-positive organisms like Staphylococcus comparable to our study. ${ }^{15-17}$

CoNS was the most common organism isolated in blood culture, i.e., (43.75\%), followed by E. coli and Pseudomonas, this is comparable to studies done by Vanitha Rani et al., ${ }^{18}$ Javeed et al., ${ }^{19}$ Jain et al., ${ }^{20}$ Rajeevan et al., ${ }^{21}$ and Shrestha et al. ${ }^{22}$

Table 1: Frequency of organisms isolated

\begin{tabular}{rlc}
\hline No. & Organisms & Frequency \\
\hline 1 & Escherichia coli & $32(18.6 \%)$ \\
2 & Acinetobacter & $25(14.5 \%)$ \\
3 & Klebsiella & $20(11.6 \%)$ \\
4 & Pseudomonas & $17(9.8 \%)$ \\
5 & Coagulase negative Staphylococcus & $27(15.6 \%)$ \\
6 & Enterococcus & $13(7.5 \%)$ \\
7 & Proteus & $3(1.74 \%)$ \\
8 & Staphylococcus & $2(1.16 \%)$ \\
9 & Nonfermenting gram-negative Bacillus & $3(1.74 \%)$ \\
10 & Streptococcus & $4(2.32 \%)$ \\
11 & Fungal & $26(15.11 \%)$ \\
& Total & $127(100 \%)$ \\
\hline
\end{tabular}

E. coli $(41 \%)$ was commonly isolated from urine, followed by fungal growth and Acinetobacter. In other studies such as Bajaj et al. ${ }^{23}$ and Sheth et al., ${ }^{24}$ Klebsiella was commonly isolated from urine culture. Fungal urinary tract infection has become a significant nosocomial problem over the past decade; ${ }^{21}$ however, laboratory yield of yeast in urine and its significance may be difficult to differentiate from colonization and infection. ${ }^{24-27}$

Klebsiella was commonly isolated from ET aspirate culture (27.5\%) followed by Acinetobacter and Pseudomonas. In most other studies done in respiratory ICU, Acinetobacter was commonly isolated followed by Klebsiella and Pseudomonas. ${ }^{28-30}$

E. coli showed highest resistance to ceftazidime, cefepime, and ceftriaxone (62.5\%). This was identical to the study by Hsu et al., ${ }^{31}$ Mangaiarkkarasi et al., ${ }^{32}$ and Oteo et al. (Fig. 8). ${ }^{33}$

Acinetobacter showed high resistance to cephalosporins (96\%) followed by piperacillin-tazobactam (84\%) as also reported by Chakraverti et al. (Fig. 9). ${ }^{14}$

Klebsiella showed high resistance to cephalosporins (65\%), amikacin, gentamicin and meropenem (60\%), imepenem (45\%), and colistin (20\%). The resistance of Klebsiella to cephalosporins was also observed in other studies by Sheth et al., ${ }^{24}$ Javeed et al. (Fig. 10). ${ }^{19}$

Pseudomonas showed the highest resistance to antipseudomonal drugs such as ceftazidime (76.4\%), piperacillin-tazobactam (64.7\%),

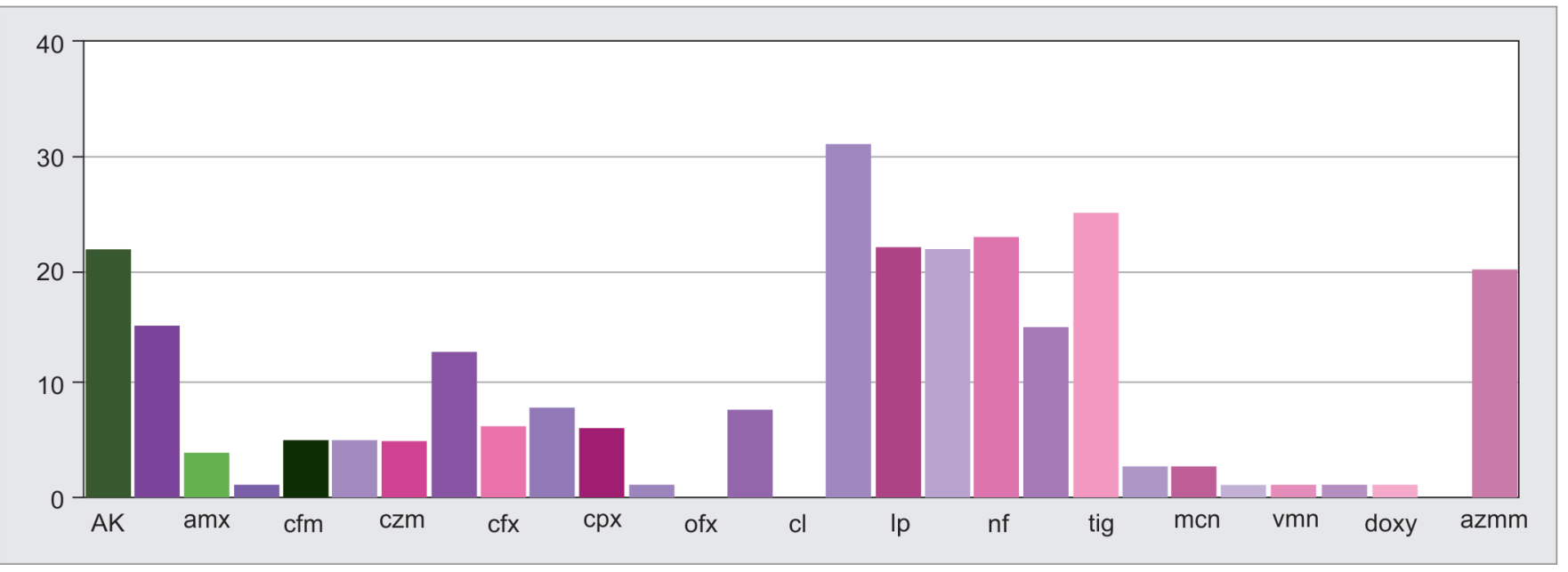

Fig. 3: Escherichia coli-sensitivity pattern

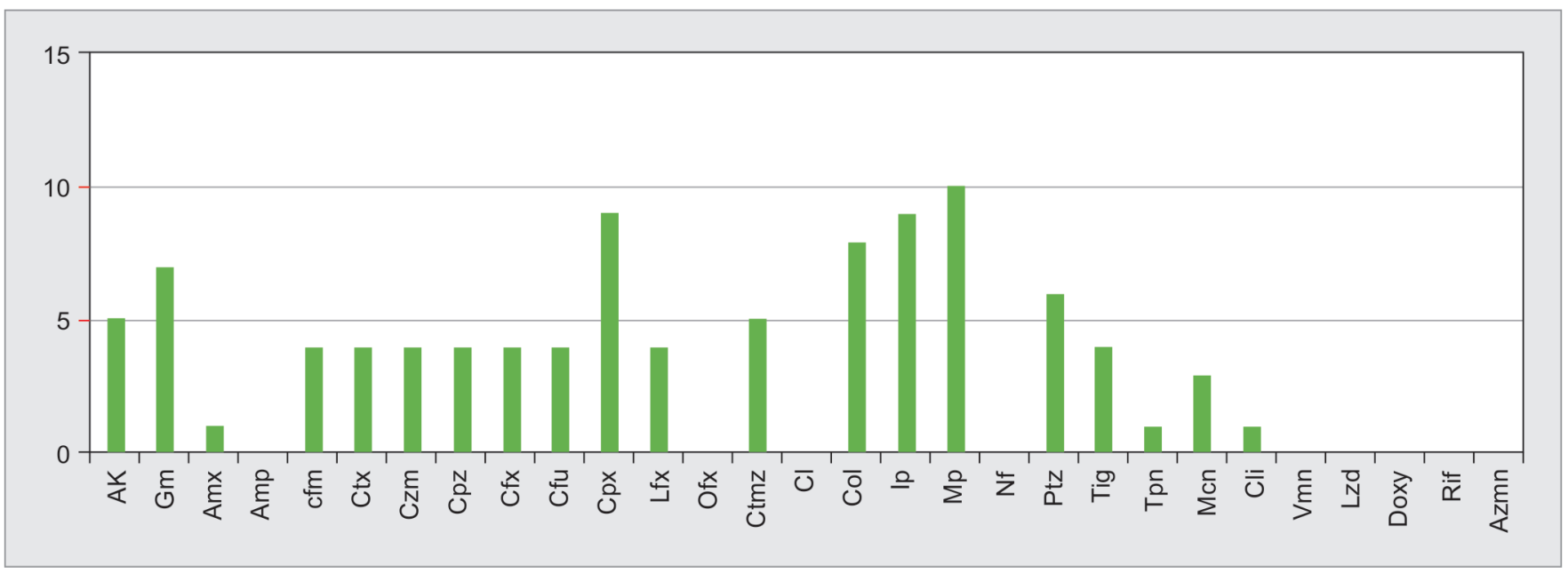

Fig. 4: Pseudomonas-sensitivity pattern 
Table 2: Antibiotic-sensitivity pattern of isolates

\begin{tabular}{|c|c|c|c|c|c|c|c|c|c|}
\hline & E. coli & Acineto & Kleb & Pseud & CoNS & Entero & Prot & Strepto & Staph \\
\hline Ak & 68.75 & 4 & 40 & 29.4 & 19.2 & 38.4 & 33.3 & 50 & 50 \\
\hline $\mathrm{Gm}$ & 46.87 & 12 & 40 & 41.1 & 50 & 23.07 & 33.3 & 75 & 50 \\
\hline Amx & 12.5 & 0 & 10 & 5.88 & 19.2 & 53.8 & 33.3 & 0 & 0 \\
\hline Amp & 3.12 & 0 & 0 & 0 & 26.9 & 0 & 0 & 0 & 0 \\
\hline $\mathrm{Cfm}$ & 15.62 & 4 & 25 & 23.5 & 26.9 & 15.3 & 0 & 75 & 50 \\
\hline Ctx & 15.62 & 4 & 25 & 23.5 & 26.9 & 15.3 & 0 & 75 & 50 \\
\hline Ctzm & 15.62 & 4 & 25 & 23.5 & 26.9 & 15.3 & 0 & 75 & 50 \\
\hline Cfpz & 40.6 & 4 & 25 & 23.5 & 26.9 & 15.3 & 0 & 75 & 50 \\
\hline Cxm & 18.7 & 4 & 25 & 23.5 & 26.9 & 15.3 & 0 & 75 & 50 \\
\hline Cfu & 25 & 4 & 25 & 23.5 & 26.9 & 15.3 & 0 & 75 & 50 \\
\hline Cpx & 18.7 & 8 & 20 & 52.9 & 30.7 & 23.07 & 0 & 25 & 50 \\
\hline Lfx & 3.12 & 0 & 0 & 23.5 & 34.6 & 0 & 0 & 0 & 0 \\
\hline Ofx & 0 & 0 & 0 & 0 & 0 & 0 & 0 & 0 & 0 \\
\hline Ctmx & 25 & 8 & 25 & 25 & 42.3 & 23.07 & 0 & 50 & 50 \\
\hline $\mathrm{Cl}$ & 0 & 0 & 0 & 0 & 42.3 & 0 & 0 & 0 & 0 \\
\hline Col & 96.8 & 68 & 70 & 47.05 & 19.2 & 23.07 & 0 & 50 & 50 \\
\hline Ip & 68.75 & 24 & 45 & 52.9 & 19.2 & 38.4 & 33.3 & 50 & 50 \\
\hline $\mathrm{Mp}$ & 68.75 & 28 & 30 & 58.8 & 23.07 & 30.7 & 33.3 & 25 & 50 \\
\hline $\mathrm{Nf}$ & 71.8 & 0 & 0 & 0 & 61.5 & 23.07 & 0 & 0 & 100 \\
\hline Ptz & 46.87 & 16 & 0 & 35.2 & 15.3 & 30.7 & 33.3 & 0 & 50 \\
\hline Tig & 78.12 & 64 & 55 & 23.5 & 69.2 & 76.9 & 0 & 25 & 100 \\
\hline Tpn & 9.37 & 0 & 15 & 5.88 & 76.9 & 61.5 & 0 & 0 & 50 \\
\hline Mcn & 9.37 & 40 & 10 & 17.6 & 57.6 & 15.3 & 0 & NT & 50 \\
\hline Cli & 3.12 & 0 & 5 & 5.88 & 57.6 & 30.7 & 0 & 75 & 0 \\
\hline Vmn & 3.12 & 0 & 5 & 0 & 61.5 & 76.9 & 0 & 75 & 50 \\
\hline Lzd & 3.12 & 0 & 0 & 0 & 57.6 & 84.6 & 33.3 & 75 & 50 \\
\hline Doxy & 3.12 & 0 & 0 & 0 & 50 & 15.3 & 0 & 0 & 50 \\
\hline Rif & 0 & 4 & 0 & 0 & 42.3 & 23.07 & 0 & 0 & 50 \\
\hline Aznm & 62.5 & 0 & 0 & 0 & 0 & 0 & 0 & 0 & 0 \\
\hline
\end{tabular}

Ak, amikacin; Amx, amoxicillin; Amp, ampicillin; Gm, gentamicin; Cfm, cefepime; Ctx, ceftriaxone; Czm, ceftazidime; Cpz, cefaperazone; Cfx, cefexime; Cfu, cefuroxime; Cpx, ciprofloxacin; Lfx, levofloxacin; Ofx, oflaxacin; Ctmz, cotrimoxazole; Cl, clarithromycin; Col, colistin; Ip, imepenem; Mp, meropenem; $\mathrm{Nf}$, nitrofurantoin; Ptz, piperacillin-tazobactam; Tig, tigecycline; Tpn, tiecoplanin; Mcn, minocycline; Cli, clindamycin; Vmn, vancomycin; Lzd, linezolid; Doxy, doxycycline; Rif, rifampicin; Aznm, aztreonam; NT, not tested; E. coli, Escherichia coli; Acineto, Acinetobacter; Kleb, Klebsiella; Pseud, Pseudomonas; Entero, Enterococcus; Prot, Proteus; Strepto, Streptococcus; Staph, Staphylococcus

The bold values indicate the rate of emergence of antibiotic resistant organisms to our basic antibiotics and need for higher antibiotics and also some multidrug resistant organisms. Its time to stay Alert!!!

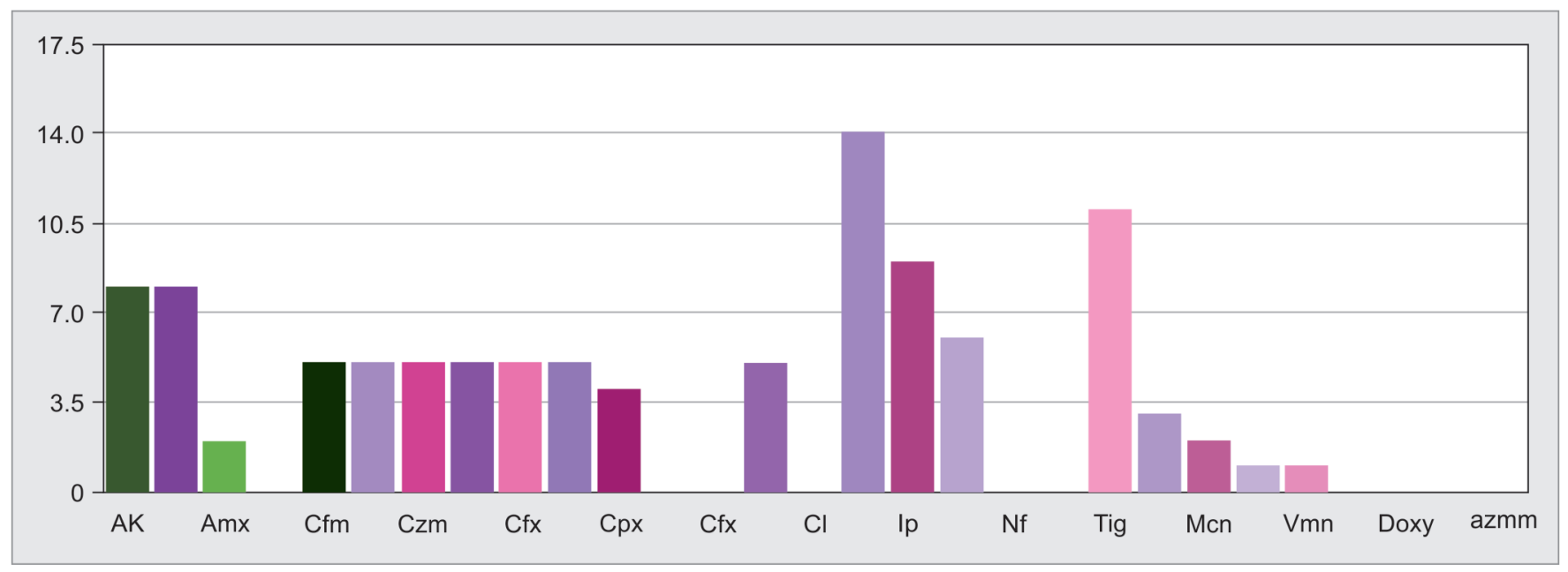

Fig. 5: Klebsiella-sensitivity pattern 
Table 3: Antibiotic-resistance pattern of isolates

\begin{tabular}{|c|c|c|c|c|c|c|c|c|c|}
\hline & E. coli & Acineto & Kleb & Pseudo & CoNS & Entero & Proteus & Strepto & Staph \\
\hline Ak & 18.75 & 0 & 60 & 70.5 & 80.7 & 61.5 & 66.6 & 50 & 50 \\
\hline $\mathrm{Gm}$ & 21.8 & 0 & 60 & 58.8 & 50 & 76.9 & 66.6 & 25 & 50 \\
\hline Amx & 37.5 & 0 & 90 & 94.1 & 80.7 & 61.5 & 66.6 & 0 & 0 \\
\hline Amp & 37.5 & 0 & 0 & 0 & 0 & 0 & 0 & 0 & 0 \\
\hline $\mathrm{Cfm}$ & 62.5 & 96 & 65 & 76.4 & 73.06 & 0 & 100 & 25 & 50 \\
\hline Ctx & 62.5 & 96 & 65 & 76.4 & 73.06 & 84.6 & 100 & 25 & 50 \\
\hline Ctzm & 62.5 & 96 & 65 & 76.4 & 73.06 & 84.6 & 100 & 25 & 50 \\
\hline Cfpz & 43.7 & 96 & 65 & 76.4 & 73.06 & 84.6 & 100 & 25 & 50 \\
\hline Cxm & 56.2 & 96 & 65 & 76.4 & 73.06 & 84.6 & 100 & 25 & 50 \\
\hline Cfu & 46.8 & 96 & 65 & 76.4 & 73.06 & 84.6 & 100 & 25 & 50 \\
\hline Cpx & 21.12 & 92 & 70 & 47.05 & 69.2 & 76.9 & 66.6 & 75 & 50 \\
\hline Lfx & 15.62 & 0 & 0 & 76.4 & 57.6 & 0 & 0 & 0 & 0 \\
\hline Ofx & 15.62 & 0 & 0 & 0 & 0 & 0 & 0 & 0 & 0 \\
\hline Ctmx & 40.6 & 92 & 65 & 70.5 & 57.6 & 76.9 & 66.6 & 50 & 50 \\
\hline $\mathrm{Cl}$ & 12.5 & 0 & 0 & 0 & 57.6 & 0 & 0 & 0 & 0 \\
\hline Col & 3.12 & 32 & 20 & 52.9 & 80.7 & 76.9 & 100 & 50 & 50 \\
\hline Ip & 21.8 & 12 & 45 & 47.05 & 80.7 & 61.5 & 66.6 & 50 & 50 \\
\hline $\mathrm{Mp}$ & 18.75 & 72 & 60 & 41.1 & 73.06 & 69.2 & 66.6 & 75 & 50 \\
\hline $\mathrm{Nf}$ & 6.25 & 0 & 0 & 100 & 42.3 & 76.9 & 66.6 & 100 & 0 \\
\hline Ptz & 40.6 & 84 & 0 & 64.7 & 84.6 & 69.2 & 66.6 & 100 & 50 \\
\hline Tig & 0 & 36 & 35 & 76.4 & 30.7 & 23.07 & 100 & 75 & 0 \\
\hline Tpn & 15.62 & 0 & 75 & 94.1 & 23.07 & 38.4 & 100 & 100 & 50 \\
\hline Mcn & 0 & 60 & 0 & 82.35 & 34.6 & 84.6 & 100 & Not & 50 \\
\hline Cli & 12.5 & 0 & 0 & 100 & 34.6 & 69.2 & 100 & 25 & 100 \\
\hline Vmn & 12.5 & 0 & 85 & 94.1 & 38.4 & 23.07 & 100 & 25 & 100 \\
\hline Lzd & 12.5 & 0 & 90 & 100 & 42.3 & 15.3 & 66.6 & 25 & 50 \\
\hline Doxy & 12.5 & 0 & 90 & 100 & 50 & 84.6 & 0 & 100 & 50 \\
\hline Rif & 15.62 & 0 & 90 & 100 & 19.2 & 76.9 & 0 & 100 & 50 \\
\hline Aznm & 12.5 & 0 & 0 & 0 & 0 & 0 & 0 & 0 & 0 \\
\hline
\end{tabular}

Ak, amikacin; Amx, amoxicillin; Amp, ampicillin; Gm, gentamicin; Cfm, cefepime; Ctx, ceftriaxone; Czm, ceftazidime; Cpz, cefaperazone; Cfx, cefexime; Cfu, cefuroxime; Cpx, ciprofloxacin; Lfx, levofloxacin; Ofx, oflaxacin; Ctmz, cotrimoxazole; Cl, clarithromycin; Col, colistin; Ip, imepenem; Mp, meropenem; Nf, nitrofurantoin; Ptz, piperacillin-tazobactam; Tig, tigecycline; Tpn, tiecoplanin; Mcn, minocycline; Cli, clindamycin; Vmn, vancomycin; Lzd, linezolid; Doxy, doxycycline; Rif, rifampicin; Aznm, aztreonam; NT, not tested; E. coli, Escherichia coli; Acinito, Acinetobacter; Kleb, Klebsiella; Pseud, Pseudomonas; Entero, Enterococcus; Prot, Proteus; Strepto, Streptococcus; Staph, Staphylococcus

The bold values indicate the rate of emergence of antibiotic resistant organisms to our basic antibiotics and need for higher antibiotics and also some multidrug resistant organisms. Its time to stay Alert!!!

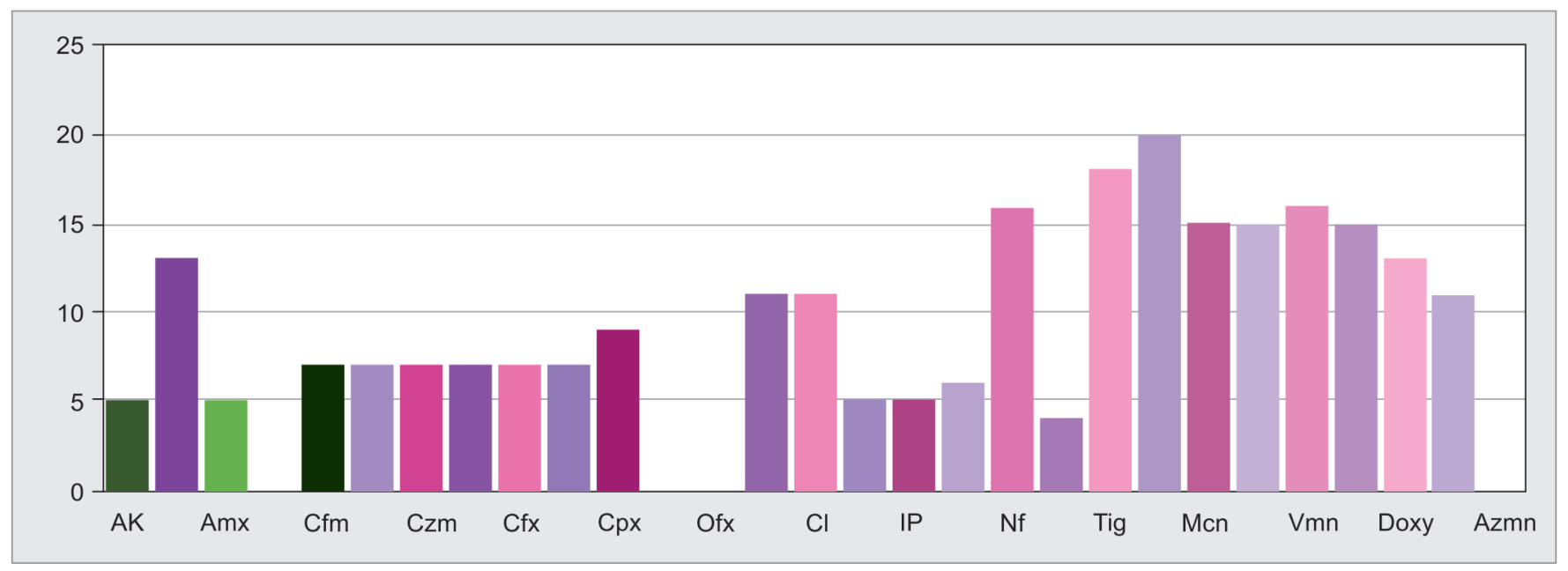

Fig. 6: CoNS-sensitivity pattern 


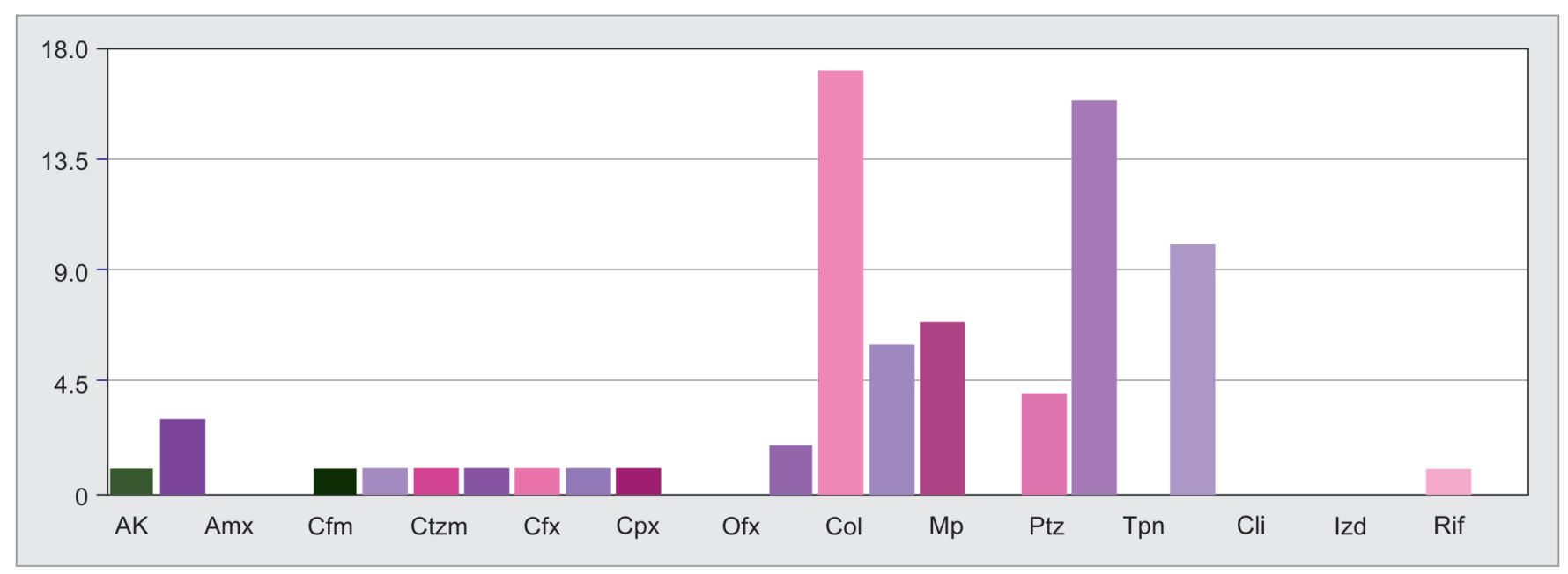

Fig. 7: Acinetobacter-sensitivity pattern

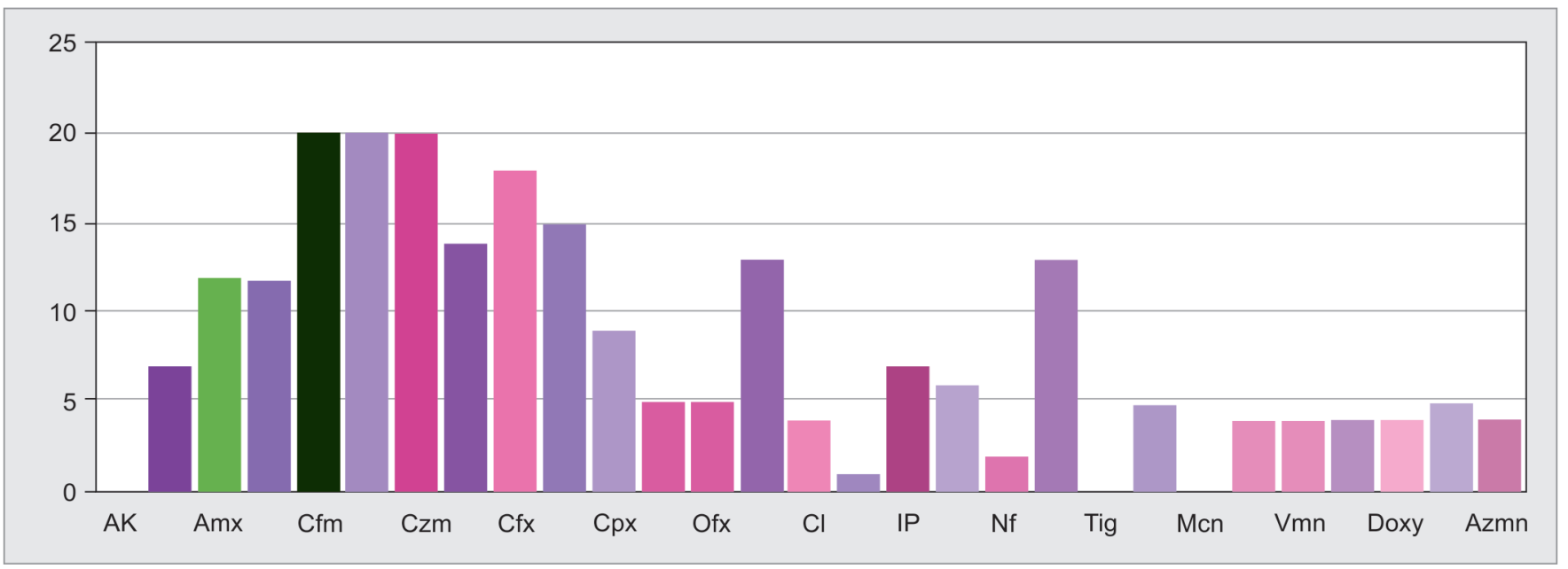

Fig. 8: Escherichia coli-resistance pattern

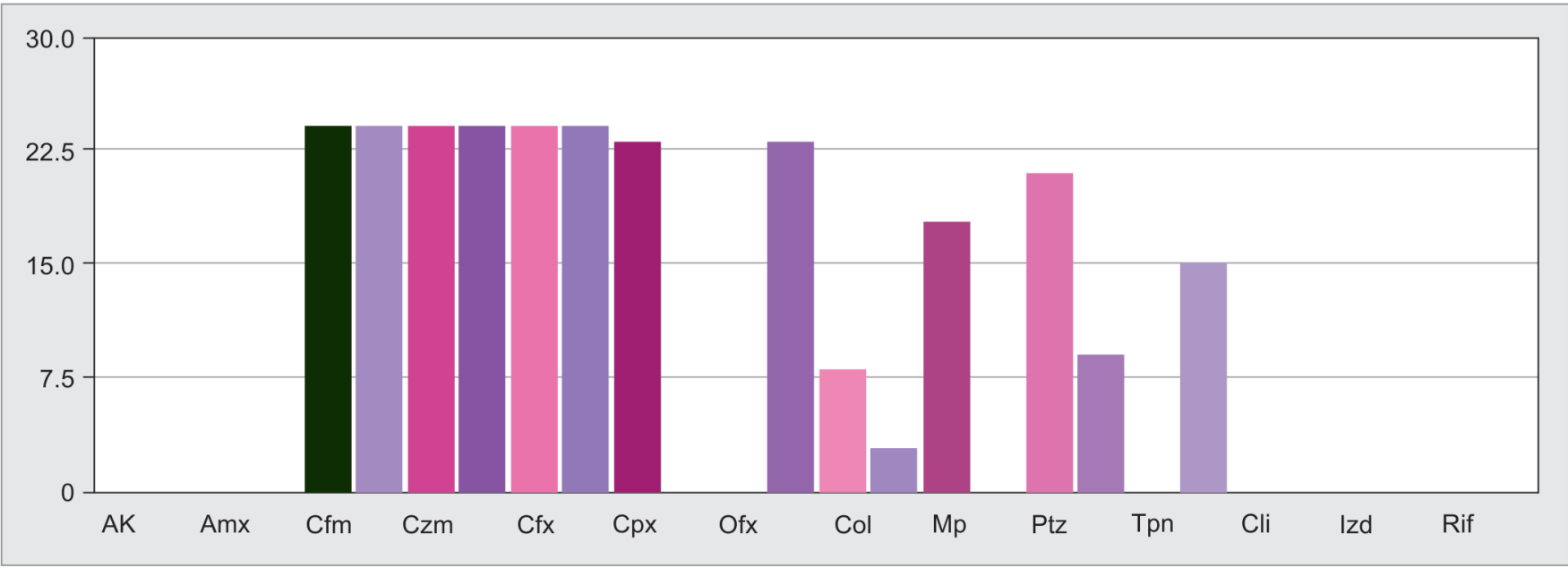

Fig. 9: Acinetobacter-resistance pattern

amikacin (70.5\%), gentamicin (58\%), imepenem (47\%), and meropenem (41.1\%). It also showed high resistance to colistin, i.e., (52\%), this pattern of resistance was observed by Mohanasundaram et al. ${ }^{34}$ (Fig. 11).
Enterococcus showed highest resistance to cephalosporins (84.6\%), amoxcillin (61\%), cotrimoxazole, and colistin (76\%). Streptococcus showed $100 \%$ resistance to piperacillin-tazobactam 


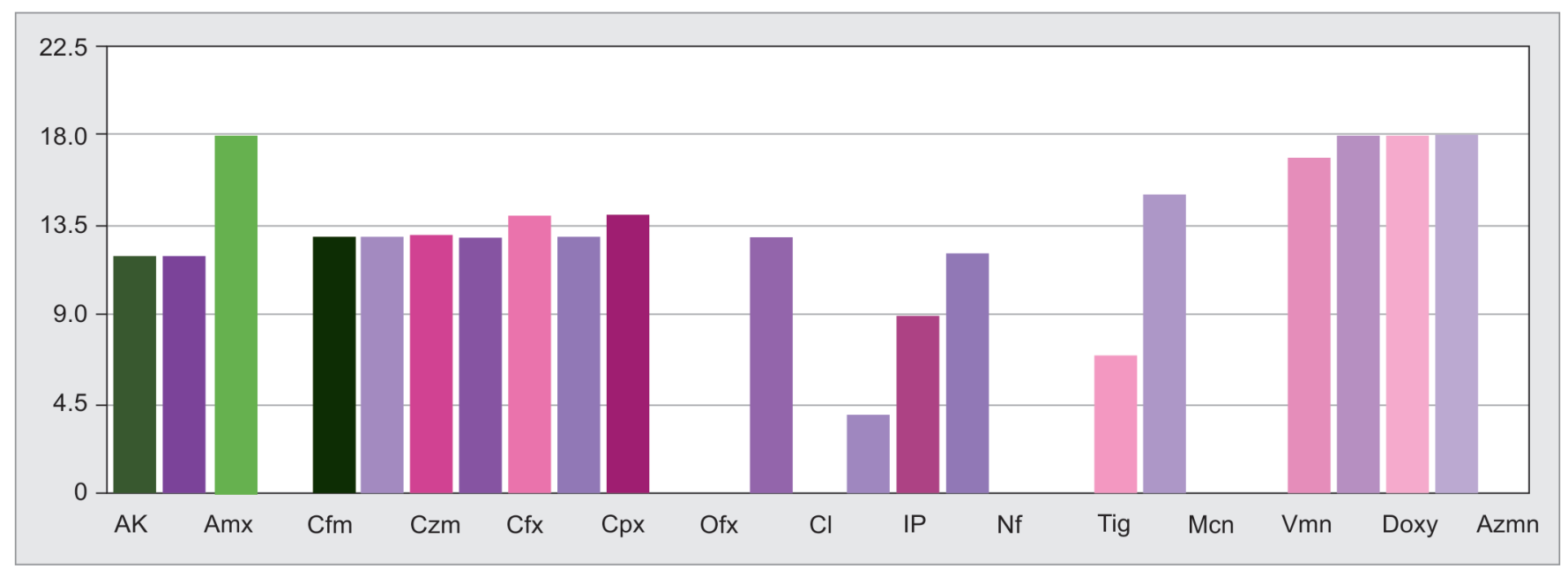

Fig. 10: Klebsiella-resistance pattern

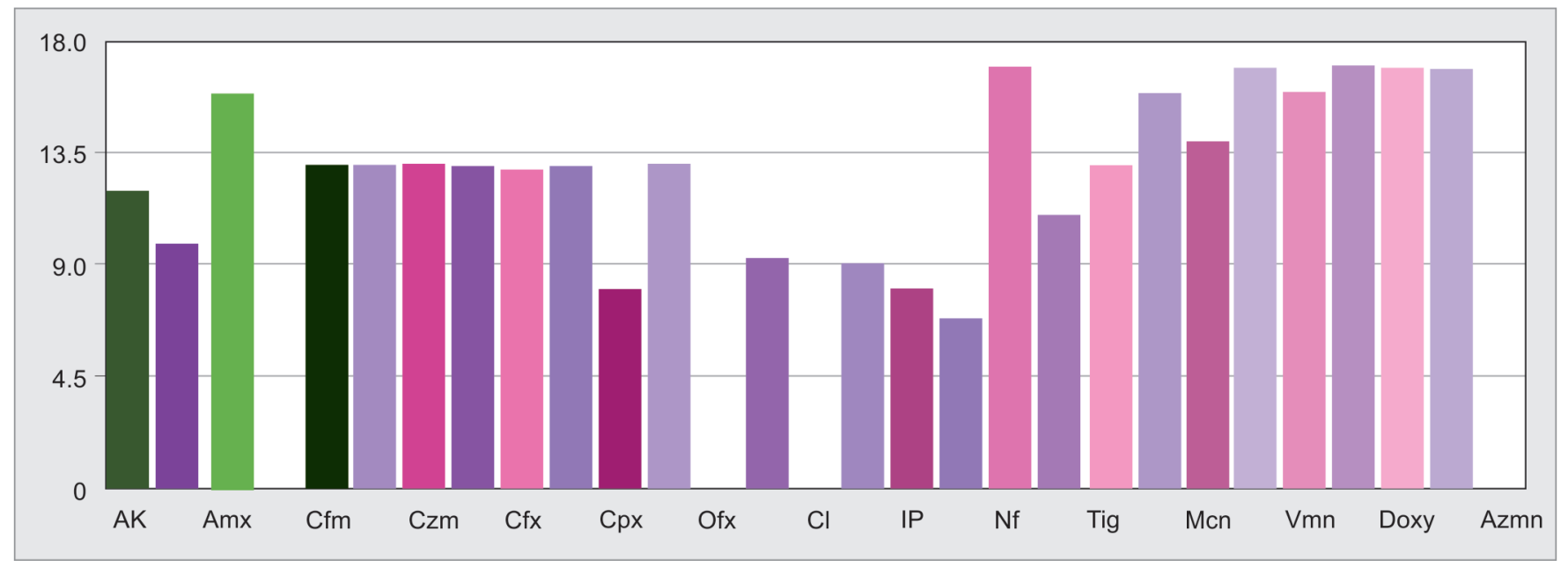

Fig. 11: Pseudomonas-resistance pattern

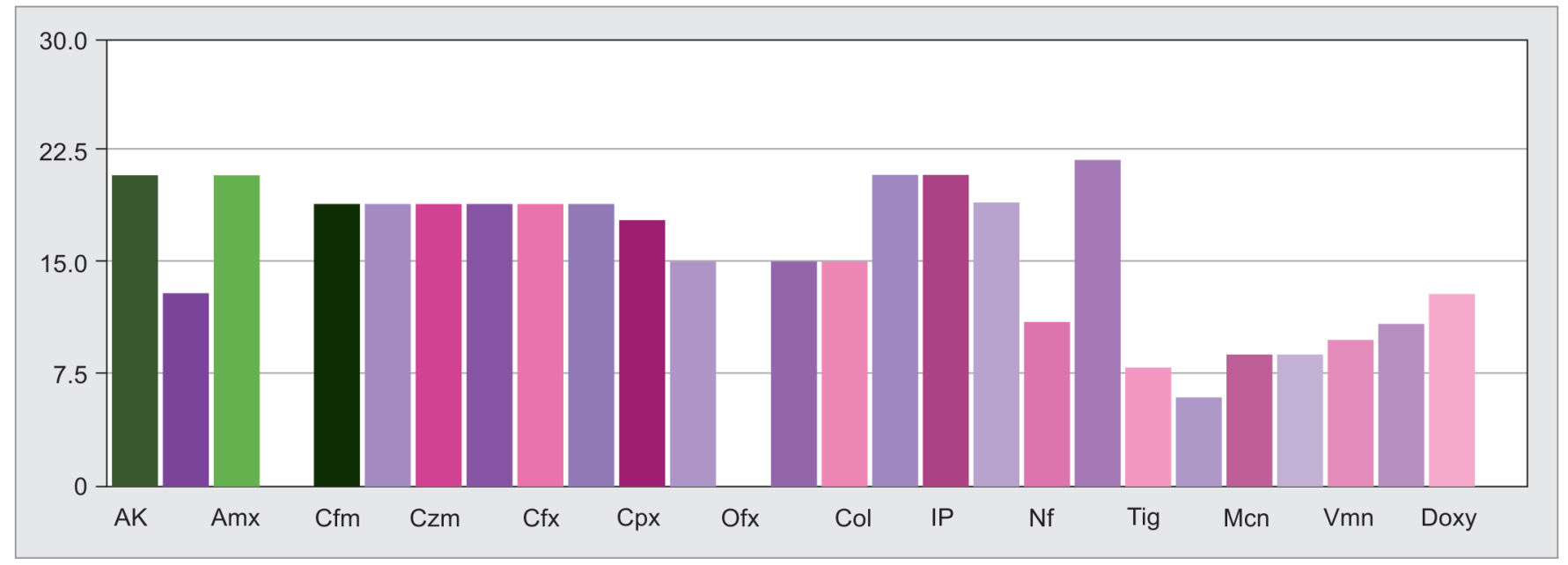

Fig. 12: CoNS-resistance pattern

and teicoplanin. Staphylococcus showed $100 \%$ resistance to vancomycin and clindamycin (Fig. 12).

In our study the most common organisms isolated from patients in ICU were gram-negative isolates such as
E. coli, Acinetobacter, Klebsiella, Pseudomonas, and Proteus which showed highest resistance to second-and thirdgeneration cephalosporins followed by piperacillintazobactam. 
Piperacillin-tazobactam has been the mainstay of empirical antibiotic therapy followed by carbapenems in severely ill ICU patients. Indian guidelines by Indian Council of Medical Research (ICMR) also recommend the use of $\beta$-lactam with $\beta$-lactamase inhibitor such as piperacillin-tazobactam as empirical antibiotic therapy in critically ill patients. In our study, we observed significantly high resistance to piperacillin and tazobactum, i.e., around $40-80 \%$ in both gram-negative and positive infections, in the obtained culture and sensitivity reports.

Carbapenem-resistant Enterobacteriaceae including Klebsiella, E. coli, and Acinetobacter has emerged with increasing prevalence over the past decade, which is also evident in our study where E. coli showed around $68 \%$ sensitivity to carbapenems, whereas Acinetobacter showed only 24\%, Klebsiella 30-45\%, and Pseudomonas 50-55\%.

This may be due to the prior antibiotic usage, prior severe gram-negative infections, inappropriate course of antibiotics, and patients coming with severe sepsis and septic shock as ours is a tertiary care hospital.

With the emergence of these multidrug-resistance organisms, older medications such as colistin has been revived. Even in our study, we observed good sensitivity of gram-negative isolates to colistin, where E. coli showed $96.8 \%$ sensitivity, Acinetobacter $68 \%$, Klebsiella 70\%, and Pseudomonas 47\%.

But few pan-drug-resistant isolates were also identified in our study, which were resistant to all drugs including carbapenems, colistin, and minocycline. Emergence of such pan-drug-resistant organisms are threat to mankind and do makes us think what next.

Probably at this stage, a local antibiogram has to be drawn in every ICU setup, at least quarterly, for better clinical decisionmaking regarding initiation of empirical antibiotics with antibiotic stewardship program, which are beneficial in preventing the emergence of MDR and extremely drug resistant organisms. Most important in this is the use of broad-spectrum empirical antimicrobials with an aggressive de-escalation strategies to minimize collateral damage to current and future patients. Emphasis should also be laid on the use of sterile techniques while inserting devices, hand hygiene and use of gowns and gloves in ICU to prevent nosocomial infections and better patient response and clinical outcome.

\section{Conclusion}

Antibiotic resistance is a major upcoming problem in today's clinical practice, increasing the challenges to treating consultants as well as huge financial burden to patient bystanders. Gram-negativeresistant infections are increasing in our ICU setups, leading to increased morbidity and mortality. Hence, timely antibiogram and antibiotic stewardship programs have to be conducted for a better understanding of the type of organism, their sensitivity and resistance pattern, so as to initiate empirical antibiotics in emergency conditions. Also equal emphasis has to be given for de-escalation of antibiotics whenever indicated, so as to prevent further misuse of antibiotics and increase the resistance of these organisms. Better usage of available drugs lead to better preservation of stores for future generation.

\section{References}

1. World Health Organisation (WHO), WAAW: World Antibiotic Awareness Week, 2018, available from: https://www.who.int/ campaigns/world-antibiotic-awareness-week/world-antibioticawareness-week-2018.
2. Travasso C. India draws a red line under antibiotic misuse. BMJ 2016;352:11202. DOI: 10.1136/bmj.i1202.

3. World Health Organization (WHO). Antimicrobial resistance: draft global action plan on antimicrobial resistance, 2015, available from: http://www.wpro.who.int/entity/drug_resistance/resources/ global_action_plan_eng.pdf.

4. Ganguly NK, Arora NK, Chandy SJ, Fairoze MN, Gill JP, Gupta U, et al. Rationalizing antibiotic use to limit antibiotic resistance in India. Indian J Med Res 2011;134:281-294.

5. Magill SS, Edwards JR, Bamberg W, Beldavs ZG, Dumyati G, Kainer MA, etal. Multistate point-prevalence survey of health care-associated infections. N Engl J Med 2014;370(13):1198-1208. DOI: 10.1056/NEJMoa1306801.

6. Prabaker K, Weinstein RA. Trends in antimicrobial resistance in intensive care units in the United States. Curr Opin Crit Care 2011;17(5):472-479. DOI: 10.1097/MCC.0b013e32834a4b03.

7. de Kraker ME, Davey PG, Grundmann H, BURDEN study group. Mortality and hospital stay associated with resistant Staphylococcus aureus and Escherichia coli bacteremia: estimating the burden of antibiotic resistance in Europe. PLoS Med 2011;8(10):e1001104. DOI: 10.1371/journal.pmed.1001104.

8. Sader HS, Flamm RK, Jones RN. Tigecycline activity tested against antimicrobial resistant surveillance subsets of clinical bacteria collected worldwide (2011). Diagn Microbiol Infect Dis 2013;76(2): 217-221. DOI: 10.1016/j.diagmicrobio.2013.02.009.

9. Bouchillon SK, Badal RE, Hoban DJ, Hawser SP. Antimicrobial susceptibility of inpatient urinary tract isolates of gram-negative bacilli in the United States: results from the study for monitoring antimicrobial resistance trends (SMART) program: 2009-2011. Clin Ther 2013;35(6):872-877. DOI: 10.1016/j.clinthera.2013.03.022.

10. Paterson DL, Ko WC, Von Gottberg A, Mohapatra S, Casellas JM, Goossens $\mathrm{H}$, et al. Antibiotic therapy for Klebsiellapneumoniae bacteremia: implications of production of extended-spectrum betalactamases. Clin Infect Dis 2004;39(1):31-37. DOI: 10.1086/420816.

11. Trouillet JL, Chester J, Vuagnat A, Joly-Guillou ML, Combaux D, Dombret MC, et al. Ventilator associated pneumonia caused by potentially drug-resistant bacteria. Am J Respir Crit Care Med 1998;157(2):531-539. DOI: 10.1164/ajrccm.157.2.9705064.

12. Tiwari HK, Sapkota D, Das AK, Sen MR. Assesment of different tests to detect methicillin resistant Staphylococcus aureus. Southeast Asian J Trop Med Public Health 2009;40(4):801-806.

13. Radji M, Fauziah S, Aribinuko N. Antibiotic sensitivity pattern of bacterial pathogens in the intensive care unit of Fatmavati Hospital, Indonesia. Asian Pac J Trop Biomed 2011;1(1):39-42. DOI: 10.1016/ S2221-1691(11)60065-8.

14. Chakraverti TK, Tripathi PC. Pattern of antibiotic susceptibility of common isolates in ICU of a tertiary care hospital: 2 years study. Int J Clin Biomed Res 2015;1(2):79-86.

15. Lautenbach E, Synnestvedt M, Weiner MG, Bilker WB, Vo L, Schein J, et al. Imipenem resistance in Pseudomonas aeruginosa: emergence, epidemiology, and impact on clinical and economic outcomes. Infect Control Hosp Epidemiol 2010;31(1):47-53. DOI: 10.1086/649021.

16. Lautenbach E, Polk RE. Resistant gram-negative bacilli: a neglected healthcare crisis? Am J Health Syst Pharm 2007;64(23 suppl 14): S3-S21; quiz S22-S24. DOI: 10.2146/ajhp070477.

17. Dellit TH, Owens RC, McGowan Jr JE, Gerding DN, Weinstein RA, Burke $J P$, et al. Infectious Diseases Society of America and the Society for Healthcare Epidemiology of America guidelines for developing an institutional program to enhance antimicrobial stewardship. Clin Infect Dis 2007;44(2):159-177. DOI: 10.1086/510393.

18. Vanitha Rani N, Gopal K, Venkata Narendra M, Vishwakanth D, Nagesh VRD, Yogita $M$, et al. A retrospective study on blood stream infections and antibiotic susceptibility patterns in a tertiary care teaching hospital. Int J Pharm Pharm Sci 2012;4(1):543-548.

19. Javeed I, Rubeena Hafeezamd M, Anwar S. Antibiotic susceptibility pattern of bacterial isolates from patients admitted to a tertiary care hospital in Lahore. Biomedica 2011;27(18):19-23.

20. Jain A, Agarwal A, Verma RK, Awasthi S, Sing KP. Intravenous device associated blood stream staphylococcal infection in 
paediatric patients. Indian J Med Res 2011;134:193-199. DOI: 10.4103/0971-5916.92642.

21. Rajeevan S, Ahmad SM, Jasmin PT. Study of prevalence and antimicrobial susceptibility pattern in blood isolates from a tertiary care hospital in North Kerala, India. Int J Curr Microbiol Appl Sci 2014;3(4):655-662.

22. Shrestha S, Shrestha NC, Dongol Singh S, Shrestha RPB, Kayestha S, Shrestha $M$, et al. Bacterial isolates and its antibiotic susceptibility pattern in NICU. Kathmandu Univ Med J (KUMJ) 2013;11(41):66-70. DOI: $10.3126 /$ kumj.v11i1.11030.

23. Bajaj JK, Karyakarte RP, Kulkarni JD, Deshmukh AB. Changing aetiology of urinary tract infections and emergence of drug resistance as a major problem. J Commun Dis 1999;31(3):181-184.

24. Sheth KV, Patel TK, Malek S, Tripathi CR. Antibiotic sensitivity pattern of bacterial isolates from the ICU of a tertiary care hospital in India. Trop J Pharm Res 2012;11(6):991-999.

25. Beck-Sagué C, Jarvis WR. Secular trends in the epidemiology of nosocomial fungal infections in the United States, 1980-1990. National Nosocomial Infections Surveillance System. J Infect Dis 1993;167(5):1247-1251. DOI: 10.1093/infdis/167.5.1247.

26. Storefront SP, Medoff G, Fraser VJ, Powderly WG, Duncan WC. Candiduria: retrospective review in hospitalised patients. Infect Dis Clin Pract 1994;3:23-29. DOI: 10.1097/00019048-199401000-00004.

27. Schönebeck J, Ånséhn S. The occurrence of yeast-like fungi in the urine under normal conditions and in various types of urinary tract pathology. Scand J Urol Nephrol 1972;6(2):123-128. DOI: 10.3109/00365597209133624.
28. Agarwal R, Gupta D, Raif P, Aggarwal AN, Jindal SK. Epidemiology, risk factors and outcome of nosocomial infection in respiratory intensive care unit in North India. J Infect 2006;53(2):98-105. DOI: 10.1016/j. jinf.2005.10.021.

29. Prashanth K, Badrinath S. Nosocomial infections due to Acinetobacter species: clinical findings, risk and prognostic factors. Indian J Med Microbiol 2006;24(1):39-44. DOI: 10.4103/0255-0857.19893.

30. Ghanshani R, Gupta R, Gupta BS, Kalra S, Khedar RS, Sood S, et al. Epidemiological study of prevalence, determinants, and outcomes of infections in medical ICU at a tertiary care hospital in India. Lung India 2015;32(5):441-448. DOI: 10.4103/09702113.164155.

31. Hsu L-Y, Tan T-Y, Jureen R, Koh T-H, Krishnan P, Tzer-Pin Lin R, et al. Antimicrobial drug resistance in Singapore hospitals. Emerg Infect Dis 2007;13(12):1944-1947. DOI: 10.3201/eid1312.070299.

32. Mangaiarkkarasi A, Meher Ali R, Gopal R. Bacteriological profile of gram negative organisms and drug sensitivity pattern of escherichia coli in hospital specimens. Int J Recent Sci Res 2013;4(5):572-575.

33. Oteo J, Campos J, Baquero F. Antibiotic resistant in 1962 invasive isolates of Escherichia coli in 27 Spanish hospitals participating in the participating in the European antimicrobial resistance surveillance system. J Antimicrob Chemother 2002;50(6):945-952. DOI: 10.1093/ jac/dkf255.

34. Mohanasundaram KM. The antimicrobial resistance pattern in the clinical isolates of Pseudomonas aeruginosa in a tertiary care hospital. J Clin Diagn Res 2011;5(3):491-494. 